\title{
Long-Term Survival of Patients With Idiopathic Inflammatory Myopathies According to Clinical Features A Longitudinal Study of 162 Cases
}

\author{
Katalin Dankó, MD, PhD, Andrea Ponyi, MD, Tamás Constantin, MD, Gábor Borgulya, MD, MSc, \\ and Gyula Szegedi, MD, DSc
}

\begin{abstract}
The idiopathic inflammatory myopathies are characterized by chronic muscle inflammation and involvement of internal organs, which contribute considerably to the morbidity and mortality of the disease. We conducted the current study to determine the survival data for patients with idiopathic inflammatory myopathies according to the presence of extramuscular clinical manifestations. We also determined the cumulative survival probability and the long-term prognosis and analyzed the causes of death at a single clinical immunology center.

A survival analysis was performed using data for 162 patients diagnosed between 1976 and 1997 according to Bohan and Peter's criteria. Patients were followed up for a minimum of 5 years (median, $101.5 \mathrm{mo}$ ) or to date of death. Cumulative survival probability was calculated by the Kaplan-Meier method. The influence of extraskeletal and extramuscular involvement was analyzed as prognostic factors for death by Cox proportional hazards survival model.

Eighteen disease-specific deaths occurred; pulmonary and cardiac complications were the most frequent causes of death. Global survival rates were $95 \%, 92 \%$, and $89 \%$ for 1,5 , and 10 years, respectively. Analysis for clinicopathologic subgroups revealed that cancer-associated myositis had the worst prognosis, while juvenile and overlap myositis had the best prognosis. Fiveand 10 -year survival rates were $94.2 \%$ and $89.4 \%$ for patients with primary polymyositis and $90.1 \%$ and $86.4 \%$ for primary dermatomyositis patients, respectively. In the whole group of patients with idiopathic inflammatory myopathy, cardiac $(\mathrm{p}<0.01)$ and respiratory muscle involvement $(\mathrm{p}=0.045)$ were significant prognostic factors for death. In the group of patients with primary
\end{abstract}

From the 3rd Department of Internal Medicine, Division of Clinical Immunology, Medical and Health Science Center, University of Debrecen (KD, AP, GS), Debrecen; and the 2nd Department of Pediatrics, Semmelweis University, Faculty of Medicine (TC, GB), Budapest, Hungary.

Address reprint requests to: Katalin Dankó, MD, PhD, 3rd Department of Internal Medicine, Division of Clinical Immunology, Medical and Health Science Center, University of Debrecen 4004 Debrecen, Móricz Zs Krt 22, Hungary. E-mail: danko@iiibel.dote.hu.

Copyright (C) 2004 by Lippincott Williams \& Wilkins

ISSN: 0025-7974/04/8301-0035

DOI: $10.1097 / 01 . m d .0000109755 .65914 .5 \mathrm{e}$ polymyositis/dermatomyositis, cardiac involvement was the main prognostic factor for death $(\mathrm{p}<0.01)$.

Myositis patients described in this study have higher survival rates than reported previously worldwide. We examine the reasons for the differences between the data in the current study and the available survival data in the relevant literature.

(Medicine 2004;83:35-42)

Abbreviations: $\mathrm{DM}=$ dermatomyositis, $\mathrm{IIM}=$ idiopathic inflammatory myopathy, $\mathrm{PM}=$ polymyositis.

\section{INTRODUCTION}

$\mathrm{T}_{\mathrm{se}}^{\mathrm{h}}$ he idiopathic inflammatory myopathies (IIMs) are systemic autoimmune diseases characterized by chronic muscle inflammation resulting in progressive weakness and frequent involvement of internal organs, mainly the pulmonary, gastrointestinal, and cardiac systems, which contribute considerably to the morbidity and mortality of the IIMs. Mortality rate was $50 \%$ in a year for the most frequent myopathies, polymyositis (PM) and dermatomyositis (DM), before the widespread use of glucocorticoids ${ }^{1}$. In the past decades, earlier diagnosis and adequate treatment regiments have become the standard of care, so survival of patients with IIMs has improved progressively worldwide ${ }^{10,18}$.

We report here a long-term study of 162 Hungarian patients who were followed at a single clinical immunology center with a particular interest in the disease and which is responsible for the health provision of about 2.5 million people. Our series represents most of the types of IIMs defined by Bohan and Peter. To our knowledge, this is among the largest survival studies on patients with IIMs in the world.

\section{METHODS}

\section{Patient Selection: Recruitment and Analysis Dates}

We identified 162 consecutive inpatients and outpatients who were diagnosed, treated, and followed by the 
3rd Department of Internal Medicine, University of Debrecen, Hungary. The diagnosis of IIM was made between 1 January 1976 and 31 December 1997. We analyzed the medical records retrospectively and recorded the following data: age, sex, time of diagnosis, duration of symptoms before the diagnosis was made, extraskeletal and extramuscular manifestations at any time during the clinical course, and date of death or end of follow-up. All patients were white.

\section{Diagnosis}

Diagnosis of IIM was based on the criteria defined by Bohan and Peter $^{3}$ in all cases:

1. Progressive, symmetric weakness of proximal muscles;

2. Elevation of creatinine-kinase, lactate acid dehydrogenase;

3. Characteristic triad of electromyographic alterations;

4. Muscle biopsy evidence;

5. Characteristic dermatologic features in DM.

Patients were classified into clinicopathologic subgroups according to the Bohan and Peter schema as follows:

I. Primary, adult PM;

II. Primary, adult DM;

III. Juvenile idiopathic inflammatory myositis: below 18 years old at onset of disease, both DM and PM;

IV. Cancer-associated myositis;

V. Overlap myositis: myositis associated with all or some clinical and immunologic features of another systemic autoimmune disease (fulfill partly or completely the diagnostic criteria of another connective tissue disease).

\section{Clinical Evaluation}

At the time of diagnosis all patients underwent a standardized clinical evaluation to detect extramuscular (Raynaud phenomenon, interstitial lung disease) and extraskeletal (dysphagia, cardiac and respiratory muscle involvement) manifestations:

1. Chest radiograph, pulmonary function tests, highresolution computerized tomography (CT) scan of the lungs;

2. Electrocardiogram, echocardiography, Holter monitoring;

3. Dysphagia - clinical history of disordered swallowing, requirement for nasogastric tube;

4. Nailfold capillary microscopy.

During the clinical course of the disease, these tests were usually repeated annually or as required (for example, if a relapse occurred).

Systemic involvement was defined as follows:

1. Interstitial lung disease was considered if chest radiograph and/or high-resolution CT scan indicated the presence of bibasilar interstitial fibrosis or alveolar infiltrates; with pulmonary function tests abnormalities characterized by a restrictive pattern.
2. Diagnosis of cardiac involvement was based on the exclusion of other causes of rhythm disturbances, conduction defects, myocarditis, cardiomyopathy, and congestive heart failure. In some cases ( 8 from the total of 15 patients with cardiac involvement), a histopathologic evaluation confirmed the presence of cardiac involvement.

3. Respiratory muscle involvement, resulting from the weakness of the respiratory musculature, was considered if patients had ventilatory failure with decreased vitalcapacity.

Diagnosis of extramuscular and extraskeletal manifestations was considered if other causes of interstitial lung disease, dysphagia, cardiac involvement, or respiratory muscle involvement were excluded.

\section{Duration of Follow-Up and Endpoints}

The data collection for this study was terminated by 1 December 2002, when the present study was performed. Duration of follow-up was determined from time 0 corresponding to the date of diagnosis to either the date of death or the date of the latest appearance at our department (endpoints). The median follow-up was 101.5 months for surviving patients (range, $5.0-312.5 \mathrm{mo}$; 25th percentile: 65.7 and 75th percentile: 144.1 ) and 47.5 month for patients who died due to IIM (range, 0.03-234.5 mo; 25th percentile: 2.3 and 75th percentile: 107.3). In the group of patients with primary $\mathrm{PM} / \mathrm{DM}$, median duration of follow-up was 96.0 months for surviving patients (range, 5.0-276.0 mo; 25th percentile: 58.7 and 75 th percentile: 144.1 ) and 59 months for patients who died due to IIM (range, 0.03-145.0 mo; 25th percentile: 1.1 and 75 th percentile: 96.0 ).

Of the 162 IIM patients, 119 patients were followed for a minimum of 5 years (except for those who had died earlier), and 32 patients (19.7\%) were lost to follow-up. Among 117 patients with primary PM/DM, 82 were followed for a minimum of 5 years (except for those who had died earlier), and 27 patients (23.1\%) were lost to follow-up. Data of these dropout patients were included in the calculation of cumulative survival probability. All of the dropout patients were in remission when we lost contact. We speculate that most of these patients were lost due to a complete recovery (24/32 patients had a monophasic disease). Therefore, the survival probability may be underestimated.

The causes of death were evaluated by autopsy or death certificate: when a patient died in our department or in another hospital, we used the date and cause of death that appeared in the autopsy record (14 patients). When the death occurred outside the hospital, we used the cause of death stated in the death certificate (6 patients).

\section{Prognostic Factors}

We selected clinical features characteristic of IIM in addition to skeletal muscle weakness. The following 
prognostic factors were considered: gender, age at time of diagnosis, presence of Raynaud phenomenon, interstitial lung disease, dysphagia, respiratory muscle involvement and cardiac involvement at any time in the clinical course. We did not analyze the effect of myositis-specific autoantibodies on survival because the tests for these autoantibodies were not available in some patients at the time of diagnosis. There were no missing values among the prognostic factors in the data set.

\section{Statistical Analysis}

Data were analyzed using SPSS for Windows 10.0 statistics software. Differences in frequencies of systemic manifestations among the clinicopathologic subgroups and correlation among the supposed prognostic factors were examined using the chi-square test. The survival curves were drawn using the Kaplan-Meier method. The log-rank test was used to determine the statistical significance of the observed differences in survival rates between patient groups. Stratified Cox regression analysis with forward stepwise variable selection method was used to assess the variables predicting death ${ }^{4}$. The number of events was insufficient to analyze the interactions between the variables - this would lead to instability in the Cox model, so we decided to discard interactions. $\mathrm{P}$ values equal to or less than 0.05 were considered significant.

\section{RESULTS}

\section{Clinical Characteristics}

Clinical characteristics of our cohort of IIM patients are presented in Table 1. Distribution of clinicopathologic subgroups revealed that the most frequent forms were PM $(46.3 \%)$ and DM (25.9\%). Age distribution exhibited the classical bimodal pattern with peaks at $10-15$ and $35-45$ years of age. The mean age at the time of diagnosis was different among the subgroups: PM and overlap myositis

TABLE 1. Characteristics of Patients with Idiopathic Inflammatory Myopathies: By Clinicopathologic Subgroup

\begin{tabular}{lcccc}
\hline & $\begin{array}{c}\text { No. of } \\
\text { Patients }\end{array}$ & \multicolumn{1}{c}{$\begin{array}{c}\text { Female: } \\
\text { Male }\end{array}$} & $\begin{array}{c}\text { Age at } \\
\text { Ratio }\end{array}$ & $\begin{array}{c}\text { Time of } \\
\text { Diagnosis (yr) }\end{array}$ \\
\hline All Patients & 162 & $(100)$ & $2.1: 1$ & $39.2 \pm 13.6$ \\
Polymyositis & 75 & $(46.3)$ & $1.8: 1$ & $39.3 \pm 11.1$ \\
Dermatomyositis & 42 & $(26.0)$ & $2.8: 1$ & $43.7 \pm 12.4$ \\
$\begin{array}{l}\text { Juvenile patients } \\
\text { Cancer/associated }\end{array}$ & 9 & $(5.5)$ & $1: 2$ & $10.4 \pm 4.4$ \\
$\quad$ myositis & 7 & $(4.3)$ & $1: 1.3$ & $48.6 \pm 18.7$ \\
Overlap myositis & 29 & $(17.9)$ & $4.8: 1$ & $39.4 \pm 10.9$ \\
\hline
\end{tabular}

patients were younger at the onset of the disease than DM and cancer-associated myositis patients. The female:male ratios were also different among the subgroups: there were larger female:male ratios in DM and overlap myositis patients than in PM patients. However, males were more predominant than females in the groups of juvenile patients and cancer-associated myositis. All cancer-associated myositis patients had DM.

Further analysis of patients with primary PM/DM revealed that most of the PM patients had definite myositis (58 patients), while 17 had probable PM. The median duration of symptoms preceding the diagnosis was 5 months (range, $0.25-120 \mathrm{mo}$ ). Among patients with DM, 40 patients were considered to have definite DM and 2 had probable DM. The median onset of muscular and/or cutaneous symptoms was 2 months (range, $0.25-24 \mathrm{mo}$ ) before the diagnosis of DM. Proximal muscle weakness was present in all patients; and in the cases of DM, all the patients showed the typical skin manifestations. Frequencies of extraskeletal and extramuscular manifestations present at any time in the clinical course are shown in Table 2. Dysphagia was significantly more frequent among DM patients $(\mathrm{p}=0.0372)$, while other systemic manifestations (cardiac and respiratory muscle involvement, interstitial lung disease, Raynaud phenomenon) were observed to a similar extent among PM and DM patients.

\section{Causes of Death}

Twenty patients died in our cohort of patients. Eighteen disease-specific deaths occurred at a mean age of $49.3 \pm 10.1$ years (range, $30-65 \mathrm{yr}$ ), after a median duration follow-up of 30 months (range, $0.03-234.5 \mathrm{mo}$ ). The global survival curve of our patients (Figure 1) shows that the mortality of myositis is higher during the first year after diagnosis than later in the course. Of those patients with disease-related death, 8 died within 12 months after the diagnosis and 7 patients died more than 5 years after the diagnosis of myositis. The female:male ratio of the deceased ( 9 female: 9 male patients) was not similar to the ratio typical of the disease. Causes of death were cardiac (11 patients), pulmonary (4 patients), and gastrointestinal (1 patient) complications and cancer (in 2 patients who had cancerassociated myositis). Pulmonary complications occurred mostly within the first 12 months, while cardiovascular complications caused death mainly after 5 years' disease duration. In the groups of primary $\mathrm{PM} / \mathrm{DM}, 13$ diseasespecific deaths were observed: 4 deaths were due to pulmonary complications (1 PM patient had severe oropharyngeal dysfunction leading to aspiration pneumonia and 3 DM patients had respiratory muscle involvement resulting in pulmonary insufficiency), 8 deaths were due to cardiovascular manifestations ( 2 cases of arrhythmia, 3 of heart failure, 2 of cardiac arrest, and 1 case of myocardial 
TABLE 2. Frequency of Extraskeletal and Extramuscular Manifestations in Patients with Idiopathic Inflammatory Myopathies

\begin{tabular}{|c|c|c|c|c|c|c|c|c|}
\hline & \multicolumn{2}{|c|}{ IIM $(n=162)$} & \multicolumn{2}{|c|}{$\operatorname{PM}(n=75)$} & \multirow[t]{2}{*}{$\mathbf{P}$} & \multicolumn{2}{|c|}{ DM $(n=42)$} & \multirow[t]{2}{*}{$\mathbf{P}$} \\
\hline & No. & $(\%)$ & No. & $(\%)$ & & No. & $(\%)$ & \\
\hline ILD & 36 & $(22.2)$ & 16 & $(21.3)$ & NS & 11 & $(26.2)$ & NS \\
\hline Dysphagia & 31 & $(19.1)$ & 7 & $(9.3)$ & NS & 15 & $(35.7)$ & 0.0372 \\
\hline Respiratory muscle involvement & 26 & $(16.0)$ & 12 & $(16.0)$ & NS & 9 & $(21.4)$ & NS \\
\hline Cardiac involvement & 15 & $(9.3)$ & 7 & $(9.3)$ & NS & 2 & $(4.7)$ & NS \\
\hline Raynaud phenomenon & 51 & $(31.5)$ & 25 & $(33.3)$ & NS & 11 & $(26.2)$ & NS \\
\hline
\end{tabular}

Abbreviations: IIM = idiopathic inflammatory myopathies; PM = polymyositis; DM = dermatomyositis; NS = not significant; ILD = interstitial lung disease.

infarction) and 1 death was due to gastrointestinal complications (1 PM patient who had toxic megacolon).

\section{Difference Among Clinicopathologic Subgroups}

Global survival rates were $95 \%, 92 \%$, and $89 \%$ for 1 , 5 , and 10 years, respectively. Survival analysis for further clinicopathologic subgroups (see Figure 1) revealed that patients with various forms of IIMs have different survival rates. None of the juvenile patients in our study died. Cancerassociated myositis patients had a survival curve significantly different from the other subgroups $(p=0.03)$. Further, although overlap myositis patients had the best prognosis, there was actually no significant difference from the other subgroups $(p=0.65)$. All 3 deaths in the overlap myositis subgroup were due to cardiovascular complications, and both of the 2 deaths in the cancer-associated myositis subgroup were the consequence of the underlying malignant disease.

Five- and 10-year survival rates were 94.2\% and $89.4 \%$ for patients with primary PM and $90.1 \%$ and $86.4 \%$ for patients with primary DM. There was no significant difference between PM and DM survival curves $(p=0.5417)$. Both the 5- and 10-year survival rates were $96 \%$ for overlap myositis patients and $71 \%$ for patients with cancer-associated myositis, respectively.

\section{Influence of Clinical Features on Survival}

The effects of different extraskeletal and extramuscular manifestations were evaluated in the group of patients with primary PM/DM (Figures 2 and 3). PM patients with dysphagia $(\mathrm{p}<0.01)$ and cardiac involvement $(\mathrm{p}<0.01)$ had significantly worse cumulative survival probability than PM patients without dysphagia or cardiac involvement. In the group of patients with DM, survival was significantly worse for male patients $(p=0.0382)$, patients who were older than 45 years at time of diagnosis $(\mathrm{p}=0.0217)$, patients with interstitial lung disease $(\mathrm{p}=0.0228)$, and patients with cardiac involvement $(\mathrm{p}<0.01)$.
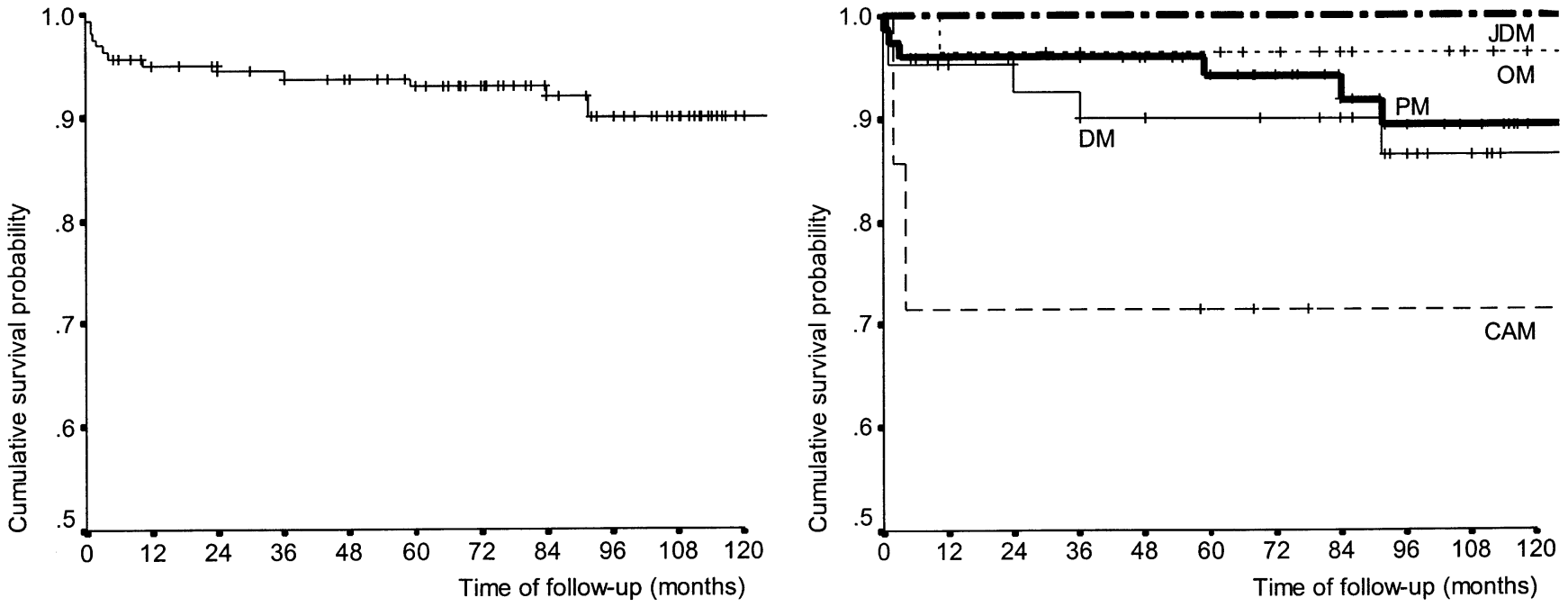

FIGURE 1. Survival curves of patients with idiopathic inflammatory myopathies (left) and survival curves according to clinicopathologic subgroups (right). JDM indicates juvenile dermatomyositis; OM, overlap myositis; PM, polymyositis; DM, dermatomyositis; CAM, cancer-associated myositis. 

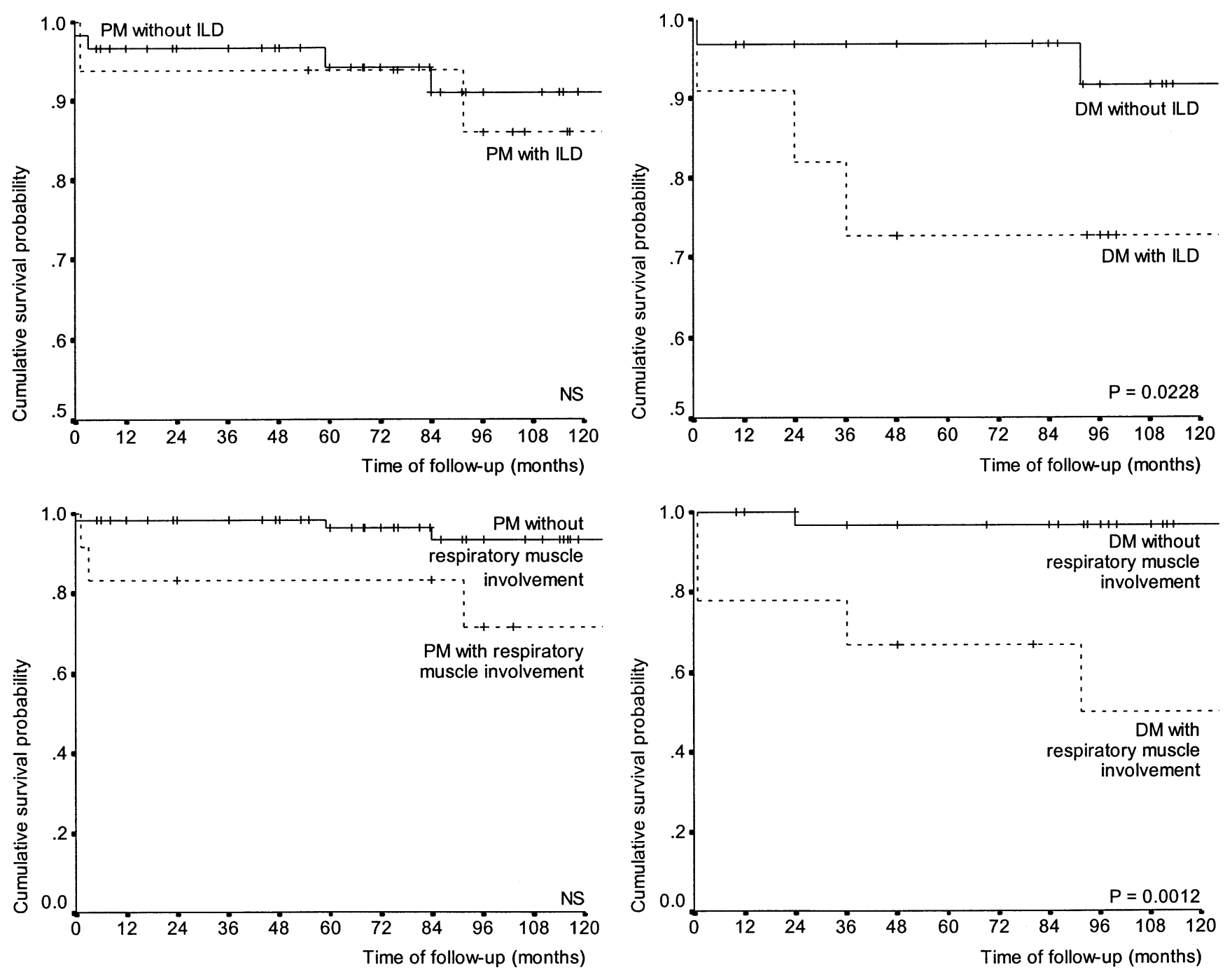

FIGURE 2. Survival curves of patients with primary polymyositis with or without interstitial lung disease (top left) and respiratory muscle involvement (bottom left); and patients with dermatomyositis with or without interstitial lung disease (top right) and respiratory muscle involvement (bottom right). PM indicates polymyositis; DM, dermatomyositis; ILD, interstitial lung disease; NS, not significant.

\section{Prognostic Factors}

Although the chi-square test of variables revealed a correlation between cardiac and respiratory muscle involvement, we analyzed the effect on survival of the presence of different clinical manifestations in a multivariate model, too. Significant prognostic factors for death determined in the whole group of patients with IIM were cardiac (coefficient $=3.182 ; \mathrm{p}<0.01$ ) and respiratory muscle involvement (coefficient $=1.16$; $\mathrm{p}=0.045$ ). In the group of patients with primary $\mathrm{PM} / \mathrm{DM}$, cardiac involvement was the main prognostic factor for death (coefficient $=3.553 ; \mathrm{p}<0.01$ ).

\section{DISCUSSION}

We report a long-term survival study on patients with myositis who were diagnosed, treated, and followed in a single clinical immunology department in Hungary. Our cohort of IIM patients represents most of the clinicopathologic forms, and their clinical characteristics (distribution of different types, female:male ratio, age distribution, and frequencies of extraskeletal and extramuscular involvement) were similar to those in other series in the literature ${ }^{10,12,17}$.

Available data on survival of patients with IIMs, based on studies with sufficient numbers of patients, come from the United States, England, France, and Israel (Table 3). The clinicopathologic subgroups of IIMs included in these studies differ considerably. Therefore, to compare our work with data of the relevant literature, we decided to report the survival probability for the whole group of IIM patients as well as for primary PM/DM patients in our series. However, survival studies are influenced by several factors that modify 

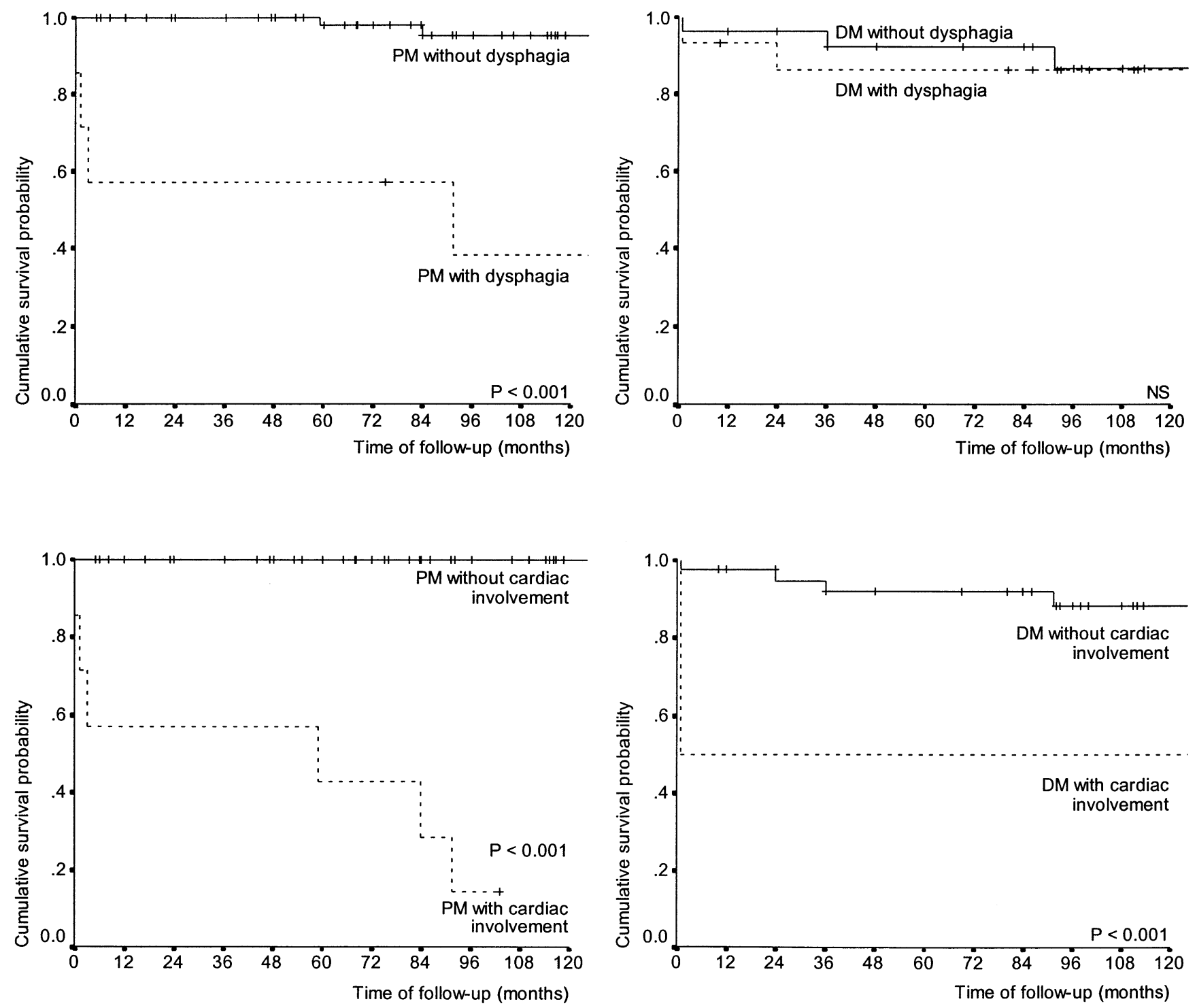

FIGURE 3. Survival curves of patients with primary polymyositis with or without dysphagia (top left) and cardiac involvement (bottom left) and patients with dermatomyositis with or without dysphagia (top right) and cardiac involvement (bottom right). PM indicates polymyositis; DM, dermatomyositis; NS, not significant.

the results considerably, and the observed differences in mortality rates are attributable to 1) the means of selection of patients (diagnostic criteria, recruitment from a single or more than 1 department, which subgroups of IIMs are included in the analysis); 2) involving a sufficient number of patients and inclusion or exclusion of data of cases lost to follow-up; and 3) the duration of follow-up.

Myositis patients in the current study had higher survival rates than previously reported worldwide. This may be attributable to 3 aspects, discussed below: 1) these large series were based on patients who were diagnosed 2-3 decades before ${ }^{2,8,14}$;2) the distribution of clinicopathologic subgroups was not similar, especially the frequency of cancer-associated cases; and 3) improved diagnostic tools and therapeutic modalities have resulted in an increase in survival rates.

First, comparison of survival data is limited by the fact that studies reported by Medsger et $\mathrm{al}^{14}$ and Hochberg et al ${ }^{8}$ had started before Bohan and Peter published their diagnostic criteria in $1975^{3}$.

Second, all of these long-term large series had different inclusion criteria, especially concerning the subgroups of IIMs they studied. The worst survival rates and prognosis were experienced in studies that involved higher rates of cancer-associated myositis patients ${ }^{2,13}$. Results of recent long-term studies of IIM patients from France and England, 
TABLE 3. Survival of Patients with Idiopathic Inflammatory Myopathies Present and Previous Reports

\begin{tabular}{lllccrrr}
\hline $\begin{array}{l}\text { First Author (Ref.) } \\
\text { Year }\end{array}$ & Location & Follow-Up & $\begin{array}{c}\text { No. of } \\
\text { Patients }\end{array}$ & $\begin{array}{c}\text { Clinicopathologic } \\
\text { Subgroup }\end{array}$ & \multicolumn{2}{c}{ Survival rates (\%) } \\
\cline { 5 - 8 } & 1-year & 5-year & $>5-$-year \\
\hline Medsger (14) 1971 & USA & $1947-1968$ & 124 & PM, DM, JDM, OM, CAM & $72 \%$ & $65 \%$ & $53 \%(7$ yr) \\
Hochberg (8) 1986 & USA & $1970-1981$ & 76 & PM, DM, JDM, OM & $94.5 \%$ & $80.4 \%$ & $72.8 \%(8$ yr) \\
Benbassat (2) 1985 & Israel & $1956-1976$ & 92 & PM, DM, JDM, OM, CAM & $72 \%$ & $52 \%$ & $82 \%$ \\
Maugars (13) 1996 & France & $1973-1984$ & 69 & PM, DM, JDM, OM, CAM & $82.6 \%$ & $66.7 \%$ & $55.4 \%(9$ yr) \\
Marie (12) 2001 & France & $1983-1998$ & 77 & PM, DM, CAM & $83 \%$ & $77 \%$ & $61 \%(15$ yr) \\
Sultan (18) 2002 & England & $1978-1999$ & 46 & PM, DM, JDM, OM & $95 \%$ & $83.8 \%(10$ yr) \\
Danko (PR) 2003 & Hungary & $1976-2002$ & 162 & PM, DM, JDM, OM, CAM & $95 \%$ & $92 \%$ & $89 \%(10$ yr) \\
\hline
\end{tabular}

Abbreviations: $\mathrm{PM}=$ polymyositis; $\mathrm{DM}=$ dermatomyositis; $\mathrm{JDM}$ = juvenile dermatomyositis; $\mathrm{OM}$ = overlap myositis; CAM = cancer-associated myositis; $\mathrm{PR}=$ present report.

which are more comparable to our experience, vary considerably depending on whether cancer-associated myositis patients are excluded or not: Sultan et $\mathrm{al}^{18}$ reported a $95 \% 5$-year survival rate, while Marie et al ${ }^{12}$ reported $77 \%$. Having different etiologic, clinical, and serologic features from the other clinicopathologic forms of IIM, cancerassociated myositis patients should not be included when measuring the cumulative survival probability and prognosis of IIM. Cancer-associated myositis patients having the worst prognosis are hardly comparable with patients with other forms of IIM, because prognosis and life expectancy is determined by the underlying malignant disease. Considering that mainly DM patients have a higher risk for malignancy ${ }^{6}$, studies including cancer-associated cases among DM patients report worse survival rates for patients with DM than for those with PM.

Finally, improved survival of patients with IIM, and other patients as well, can be attributed to the following:

1. Widespread use of new diagnostic tools and serologic testing, which contribute to early diagnosis and to recognition of milder forms of the disease;

2. Early use of more appropriate and more aggressive immunosuppressive and supportive therapy ${ }^{1}$;

3. Regular follow-up in departments specializing in the disease;

4. Better general medical care;

5. Better understanding of the natural history of the disease.

Our survival curve was heterogeneous, with an accelerated mortality during the first year after diagnosis and a slower mortality during the following 10 years, except for those who had cardiac complications later in the clinical course. We found no significant difference between groups for patients with primary PM and primary DM, although most of the studies report better survival rates for PM patients.
Major causes of death in the current study were similar to the findings of the relevant literature ${ }^{9,18}$. Deaths due to pulmonary complications were observed early in the course (median, $1 \mathrm{mo}$ ) in our cohort of patients, and cardiovascular complications occurred late in the course (median, $59 \mathrm{mo}$ ).

Myositis patients may have severe extraskeletal and extramuscular manifestations - pulmonary, gastrointestinal, and cardiac involvement-which often affect the prognosis unfavorably. In patients with primary PM, the presence of dysphagia and the presence of cardiac involvement were associated with a significantly worse survival probability. In patients with primary DM, survival rates were worsened significantly by male gender, older age at the time of diagnosis (more than $45 \mathrm{yr}$ ), presence of interstitial lung disease, and presence of cardiac involvement. Effect of pulmonary involvement, especially interstitial lung disease, on survival may be controversial ${ }^{5,7,15}$, however, most authors found higher mortality rates compared to patients without interstitial lung disease $\mathrm{e}^{2,11}$.

Classic factors of poor prognosis are older age, male sex, African-American race, interstitial lung disease, presence of anti-Jo-1 and anti-SRP autoantibodies, associated malignancy, delayed or inadequate treatment, dysphagia, dysphonia, cardiac involvement, and pulmonary involvement $^{2,9,10,13}$. In our study, unfavorable prognostic signs were respiratory muscle and cardiac involvement.

\section{ACKNOWLEDGMENT}

The authors thank Dr. Lisa Rider for critical reading of the manuscript.

\section{REFERENCES}

1. Adams EM, Plotz PH. The treatment of myositis. How to approach resistant disease. Rheum Dis Clin North Am. 1995;21:179-202.

2. Benbassat J, Geffel D, Larholt K, Sukenik S, Morgenstern U, Zlotnick A. Prognostic factors in polymyositis/dermatomyositis: a 
computer-assisted analysis of 92 cases. Arthritis Rheum. 1985;28: 249-255.

3. Bohan A, Peter BJ. Polymyositis and dermatomyositis. Parts 1 and 2 N Engl J Med. 1975;292:344-347, 403-407.

4. Cox DR. Regression models and life-tables (with discussion). J R Stat Soc B. 1972;39:86-94

5. Grau JM, Miro O, Pedrol E, Casademont J, Masanes F, Herrero C, Haussman G, Urbano-Marquez A. Interstitial lung disease related to dermatomyositis. Comparative study with patients without lung involvement. J Rheumatol. 1996;23:1921-1926.

6. Hill CL, Zhang Y, Sigurgeirsson B, Pukkala E, Mellemkjaer L, Airio A, Evans SR, Felson DT. Frequency of specific cancer types in dermatomyositis and polymyositis: a population-based study. Lancet. 2001;357:96-100.

7. Hirakata M, Nagai S. Interstitial lung disease in polymyositis and dermatomyositis. Curr Opin Rheumatol. 2000;12:501-508.

8. Hochberg MC, Feldman D, Stevens MB. Adult onset polymyositisdermatomyositis: an analysis of clinical and laboratory features and survival in 76 patients, with a review of the literature. Semin Arthritis Rheum. 1986;3:168-178.

9. Hochberg MC. Epidemiology of polymyositis/dermatomyositis. Mt Sinai J Med 1988:55:447-452.

10. Love LA, Leff RL, Fraser DD, Targoff IN, Dalakas M, Plotz PH, Miller FW. A new approach to the classification of the idiopathic inflammatory myopathy: myositis-specific autoantibodies define use- ful homogeneous patient groups. Medicine (Baltimore). 1991;70: $360-374$.

11. Marie I, Hatron P-Y, Hachulla E, Wallaert B, Michon-Pasturel U, Devulder B. Pulmonary involvement in polymyositis and dermatomyositis. J Rheumatol. 1998;25:1336-1343

12. Marie I, Hachulla E, Hatron P-Y, Hellot M-F, Levesque H, Devulder B, Courtois H. Polymyositis and dermatomyositis: short term and long term outcome, and predictive factors of prognosis. $J$ Rheumatol. 2001;28: 2230-2237.

13. Maugars YM, Berthelot J-MM, Abbas AA, Mussini J-MB, Nguyen J-MD, Prost AM. Long-term prognosis of 69 patients with dermatomyositis or polymyositis. Clin Exp Rheumatol. 1996;14:263-274.

14. Medsger TA, Robinson H, Masi AT. Factors affecting survivorship in polymyositis. A life-table study of 124 patients. Arthritis Rheum 1971;14:249-258.

15. Miro O, Laguno M, Grau JM. Survival of patients with polymyositis/ dermatomyositis and pulmonary involvement. J Rheumatol. 1999;26: $1852-1853$.

16. Rider LG, Miller FW. Idiopathic inflammatory muscle disease: clinical aspects. Baillieres Clin Rheumatol. 2000;14:37-54.

17. Spiera R, Kagen L. Extramuscular manifestations in idiopathic inflammatory myopathies. Curr Opin Rheumatol. 1998;10:556-561.

18. Sultan SM, Ioannou Y, Moss K, Isenberg DA. Outcome in patients with idiopathic inflammatory myositis: morbidity and mortality. Rheumatology. 2002;41:22-26. 
